Chemical Physics 132 (1989) 235-242

North-Holland, Amsterdam

\title{
DELAYED $S_{1} \rightarrow S_{0}$ AND $S_{2} \rightarrow S_{0}$ FLUORESCENCE, DELAYED EXCIMER FLUORESCENCE, AND PHOSPHORESCENCE FROM BIPHENYLENE
}

\author{
Joachim HERTZBERG and Bernhard NICKEL \\ Max-Planck-Institut für Biophysikalische Chemie, Abteilung Spektroskopie, Am Fassberg, D-3400 Göttingen, FRG \\ Received 7 September 1988
}

\begin{abstract}
In liquid solution, the population of the metastable triplet state of biphenylene by triplet energy transfer from a triplet sensitizer leads to different types of delayed luminescence from biphenylene: delayed $S_{1} \rightarrow S_{0}$ and $S_{2} \rightarrow S_{0}$ fluorescence and delayed excimer fluorescence due to triplet-triplet annihilation, and phosphorescence. All four delayed luminescences have been observed for the first time.
\end{abstract}

\section{Introduction}

The population of the metastable triplet state $T_{1}$ of an aromatic compound in liquid solution in general leads to different types of delayed luminescence: delayed fluorescence due to the generation of excited singlet states $\mathrm{S}_{n}(n \geqslant 1)$ by triplet-triplet annihilation (TTA) $T_{1}+T_{1} \rightarrow S_{n}+S_{0}$ and phosphorescence. In this respect the antiaromatic hydrocarbon biphenylene should not behave differently, and both types of delayed luminescence are expected to be observable. In this paper we report the first observation of different kinds of delayed fluorescence and of phosphorescence from biphenylene.

The following known facts about $T_{1}$ of biphenylene have been important for the present investigation: (a) By taking the onset of the oxygen-induced absorption band of biphenylene in chloroform for the energy $(E)$ of $\mathrm{T}_{1}, E\left(\mathrm{~T}_{1}\right) \approx h c \times 16130 \mathrm{~cm}^{-1}$ is obtained [1]. $E\left(\mathrm{~T}_{1}\right)$ cannot be much lower, since $\mathrm{T}_{1}$ of biphenylene is quenched by anthracene [2] $\left(\mathrm{T}_{1}\right.$ : $\left.14700 \mathrm{~cm}^{-1}\right)$. From this value follows that $S_{1}$ $\left(\approx 23900 \mathrm{~cm}^{-1}\right)$ and $S_{2}\left(\approx 27850 \mathrm{~cm}^{-1}\right)$ of biphenylene are accessible by TTA. (b) Since the quantum yield of intersystem crossing $S_{1} \leadsto T_{1}$ is very low, a suitable triplet sensitizer has to be used in all experimental investigations of $T_{1}$ of biphenylene [2]. (c) The decay of the sensitized triplet-triplet absorption of biphenylene at room temperature yielded a triplet lifetime $\approx 100 \mu$ s [2].
The aim of this investigation has been to detect the following delayed luminescences from biphenylene:

(1) Delayed $S_{1} \rightarrow S_{0}$ fluorescence. Because of the distinct vibrational structure of the $S_{1} \rightarrow S_{0}$ fluorescence from biphenylene, a delayed $S_{1} \rightarrow S_{0}$ fluorescence from biphenylene can be identified with certainty. Hence, also the poplation of $T_{1}$ of biphenylene as the cause of other delayed luminescences of biphenylene can be established with certainty. From the decay of the delayed $S_{1} \rightarrow S_{0}$ fluorescence a reliable value of the lifetime of $T_{1}$ is obtained.

(2) Delayed excimer fluorescence. Biphenylene forms photodimers, and an excimer has been postulated as precursor of the photodimers [3]. For the analogous photodimerization of anthracene it is known that the transformation of the excimer into the photodimer is a thermally activated process. If in this respect biphenylene behaves similarly as anthracene, then - at low temperature and low or moderate solvent viscosity - delayed excimer fluorescence from biphenylene should be observable.

(3) Delayed $S_{2} \rightarrow S_{0}$ fluorescence. In connection with the conventional estimate of the lifetime of $S_{2}$ in the preceding paper [4], the spectrum and the relative quantum yield of this delayed fluorescence have been of interest.

(4) Phosphorescence. According to Peradejordi et al. [5], the long-lived emission from a solution of biphenylene in boric acid glass [6] cannot be assigned to the phosphorescence from biphenylene. Thus the 
detection of the genuine phosphorescence from biphenylene has been of interest.

\section{Experimental}

The experimental technique has in part been described in the preceding paper [4]. In this section we report those additional experimental details which have been important for the detection of a sensitized delayed luminescence from biphenylene.

Sensitizer. The choice of a suitable triplet sensitizer has been important for the success of the present investigation. $\mathrm{N}$-methylcarbazole (NMC) [7] has been suitable on the following grounds:

(a) The triplet energy of NMC is high $\left(24250 \mathrm{~cm}^{-1}\right.$ in heptane at $193 \mathrm{~K}$ [7]). Hence the triplet energy transfer $T_{1}^{\prime}+S_{0} \rightarrow S_{0}^{\prime}+T_{1}$ is practically completely irreversible even under the most unfavourable conditions (high temperature and low relative concentration of biphenylene).

(b) The 0,0 transition of the $S_{0}^{\prime} \rightarrow S_{1}^{\prime}$ absorption band of NMC (29100 $\mathrm{cm}^{-1}$ [7]) is well above the origin of the $S_{0}, S_{2}$ absorption band of biphenylene $\left(27850 \mathrm{~cm}^{-1}\right.$ [4]). Thus the observation of a delayed $S_{2} \rightarrow S_{0}$ fluorescence from biphenylene is not impeded by the presence of NMC in a sample (due to fluorescence reabsorption ).

(c) NMC can be excited with the UV lines of an argon ion laser at $333 \mathrm{~nm}$.

(d) The quantum yield of intersystem crossing $\mathrm{S}_{1}^{\prime} \rightsquigarrow \mathrm{T}_{1}^{\prime}$ is high enough: $\phi_{\text {isc }}^{\prime} \approx 0.58$ [8].

(e) At low temperatures, a solution of biphenylene and NMC in an alkane is sufficiently photostable.

Concentrations. The choice of the concentrations $c_{0}$ of biphenylene and $c_{0}^{\prime}$ of NMC has been critical in several respects. We used $c_{0} \approx 5 \times 10^{-5} \mathrm{M}$ and $c_{0}^{\prime} \approx$ $1.0 \times 10^{-4} \mathrm{M}$.

(a) The average path length of the luminescence light in the sample has been $d \approx 0.15 \mathrm{~cm}$. This corresponds to an optical density in the maximum of the $\mathrm{S}_{0} \rightarrow \mathrm{S}_{2}$ absorption band of biphenylene (decadic molar absorption coefficient $\epsilon_{\max } \approx 1.2 \times 10^{4} \mathrm{M}^{-1} \mathrm{~cm}^{-1}$ [4]) of $\epsilon_{\max } c_{0} d \approx 0.09$, that is, to a maximal fluorescence reabsorption of $\approx 20 \%$.

(b) At the excitation wavelength $333 \mathrm{~nm}$, the absorption coefficients are $\epsilon \approx 3000 \mathrm{M}^{-1} \mathrm{~cm}^{-1}$ for biphenylene and $\epsilon^{\prime} \approx 2000 \mathrm{M}^{-1} \mathrm{~cm}^{-1}$ [7] for NMC.
Thus, at the chosen concentrations, the relative light absorption $\beta^{\prime}=\epsilon^{\prime} \mathcal{C}_{0}^{\prime} /\left(\epsilon \mathcal{C}_{0}+\epsilon^{\prime} c_{0}^{\prime}\right) \approx 0.57$ by NMC is large.

(c) The total length of the excitation light path in the fluorescence flow cell has been $1.0 \mathrm{~cm}$. For the elimination of delayed silica luminescence it has been important to measure only the light emitted from the central portion $(0.6 \mathrm{~cm})$ of the luminescent region of the solution (from $0.2 \mathrm{~cm}$ above the lower window to $0.2 \mathrm{~cm}$ below the upper window). At the given value of $\epsilon c_{0}+\epsilon^{\prime} c_{0}^{\prime} \approx 0.35 \mathrm{~cm}^{-1}$, the fraction $\alpha \approx 0.33$ of the total incident laser light is absorbed in the useful central range. This is not far from the maximal fraction $\alpha \approx 0.47$ of excitation light that can be absorbed in the observed central range, which, however, corresponds to a strongly inhomogeneous excitation in the direction of the excitation beam.

Solvent and temperature. The triplet energy transfer from NMC to biphenylene should be much faster than the triplet decay of biphenylene. Thus, in this respect a high value of $T / \eta$ is desirable, where $T$ is the temperature and $\eta$ is the viscosity. On the other hand, because of the presumably thermally activated transformation of biphenylene excimers into photodimers [3], a low temperature has been essential for the photostability of the solution. As a compromise, we chose a mixture of isopentane and cyclopentane $(7: 3)$ as solvent with low viscosity [9] and the temperature $178 \mathrm{~K}$ for the measurement of delayed fluorescence. With $\eta \approx 1.6 \times 10^{-2} \mathrm{P}$ at $178 \mathrm{~K}$ [9], the Smoluchowski-Stokes-Einstein-Debye equation yields for the rate constant of diffusion-controlled tripletenergy transfer $k_{\text {TET }} \approx \frac{8}{3} R T / \eta \approx 2.5 \times 10^{12} \mathrm{~cm}^{3}$ $\mathrm{mol}^{-1} \mathrm{~s}^{-1}=2.5 \times 10^{9} \mathrm{M}^{-1} \mathrm{~s}^{-1}$. Thus, at $c_{0}=5 \times$ $10^{-5} \mathrm{M}$, the triplet lifetime of NMC should be $\tau^{\prime} \approx$ $\left(k_{\mathrm{TET}} c_{0}\right)^{-1} \approx 8 \mu \mathrm{s}$, that is, much shorter than the triplet lifetime $\tau \approx 330 \mu$ s of biphenylene at low temperatures (cf. section 3.3).

Chopper. For the discrimination between prompt and delayed luminescences a mechanical chopper has been used. The chopper consists of two disks of 200 $\mathrm{mm}$ diameter (effective radius of $90 \mathrm{~mm}$ ) at a distance of $500 \mathrm{~mm}$ on a common shaft in a vacuumtight housing; each disk has 20 segments and runs between a pair of slits with $2 \mathrm{~mm}$ width and $6 \mathrm{~mm}$ height. The maximal rotation frequency is $500 \mathrm{~Hz}$. The experiments in the present investigation were performed with a rotation frequency of $50 \mathrm{~Hz}$, which 
corresponds to a chop frequency $f_{\mathrm{ch}}=1000 \mathrm{~Hz}$. The relation between the chop period $t_{\mathrm{ch}}=1 / f_{\mathrm{ch}}$, the excitation interval $t_{\mathrm{exc}}$, the observation interval $t_{\mathrm{obs}}$, and the deadtime $t_{\text {dead }}$ is $t_{\mathrm{ch}}=t_{\mathrm{exc}}+t_{\text {obs }}+2 t_{\text {dead }}$. In the present case we had $t_{\text {exc }}=t_{\text {obs }} \approx 375 \mu$ s and $t_{\text {dead }} \approx$ $125 \mu \mathrm{s}$. With a triplet lifetime $\tau^{\prime} \approx 8 \mu \mathrm{s}$ of NMC, the value of $t_{\text {dead }}$ corresponds to a decay of the triplet concentration of NMC by a factor of $\approx 10^{-7}$.

Excitation. The sample was excited with an argon ion laser at $333 \mathrm{~nm}$ (Spectra Physics 2035, total UV power $\approx 4 \mathrm{~W}$ ). The spectrum of the delayed fluorescence was measured with an estimated power of the incident excitation light of $\approx 0.5 \mathrm{~W}$, which corresponded to $L \approx 1.4 \times 10^{-6}$ einstein $\mathrm{s}^{-1}$. The average rate $P^{\prime}$ of production of $\mathrm{T}_{1}^{\prime}$ was $P^{\prime}=L \alpha \beta^{\prime} \phi_{\mathrm{isc}}^{\prime} / \nu$, where $v$ was the observed excited volume in which the fraction $\alpha$ of the excitation light was absorbed. With an effective laser beam diameter of $\approx 0.5 \mathrm{~mm}$, $v \approx 1.2 \mathrm{~mm}^{3}=1.2 \times 10^{-6} \mathrm{dm}^{3}$; with $\alpha=0.33, \beta^{\prime}=$ 0.57 , and $\phi_{\text {isc }}^{\prime}=0.58$, follows $P^{\prime} \approx 0.13 \mathrm{M} \mathrm{s}^{-1}$. The average steady-state triplet concentration of NMC during excitation was $\left\langle c^{\prime}\right\rangle=P^{\prime} \tau^{\prime} \approx 1.0 \times 10^{-6} \mathrm{M}$; therefore, depletion of the ground state of NMC was still small $(\approx 1 \%)$. In a similar way follows that, with a production rate $P \approx P^{\prime}$ and a lifetime $\tau \approx 330 \mu$ s (cf. section 3.3) of $T_{1}$, TTA was the dominant decay process of $T_{1}$ and depletion of the ground state of biphenylene was not negligible. Since for the triplettriplet absorption coefficient $\epsilon_{\mathrm{TT}}$ of biphenylene the relation $\epsilon_{\mathrm{TT}}(333 \mathrm{~nm}) \approx 2.5 \epsilon(333 \mathrm{~nm})$ holds [2], the depletion of the ground state of biphenylene leads to a reduction of $P^{\prime}$. Thus, neither an increase of the excitation power nor a reduction of the laser-beam diameter should lead to a marked increase of the intensity of the delayed fluorescence. The second point was verified expcrimentally. The observed intensity of the delayed fluorescence from biphenylene did not sensitively depend on the diameter of the laser beam in the sample.

Phosphorescence. For the measurement of the phosphorescence spectrum and of the triplet lifetime of biphenylene we used a photomultiplier tube with a GaAs photocathode (RCA C31034).

\section{Results and discussion}

In fig. 1 the spectrum of the delayed luminescence from a solution of biphenylene $\left(5 \times 10^{-5} \mathrm{M}\right)$ and $\mathrm{N}$ -

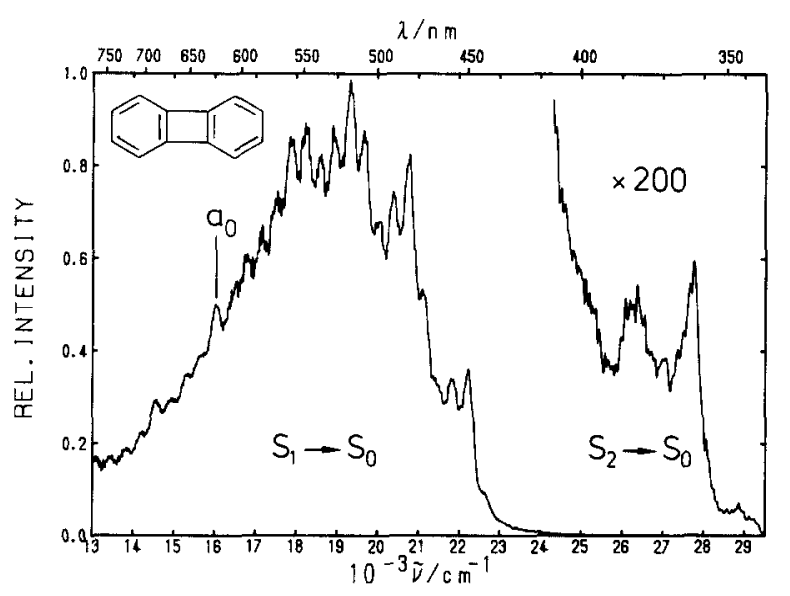

Fig. 1. Corrected spectrum of the delayed luminescence from a solution of biphenylene $\left(5 \times 10^{-5} \mathrm{M}\right)$ and $\mathrm{N}$-methylcarbazole $\left(1.0 \times 10^{-4} \mathrm{M}\right)$ in isopentane/cyclopentane at $178 \mathrm{~K}$. a denotes the false origin of the phosphorescence spectrum (cf. fig. 4 and table 1).

methylcarbazole $\left(1.0 \times 10^{-4} \mathrm{M}\right)$ in isopentane/cyclopentane at $178 \mathrm{~K}$ is shown. Two assigments are easy to make. Firstly, the comparison of this spectrum with the spectrum of the prompt fluorescence from biphenylene in 3-methylpentane at $130 \mathrm{~K}$ [4] reveals that obviously the delayed $S_{1} \rightarrow S_{0}$ fluorescence from biphenylene is the dominant component of the delayed luminescence. Secondly, the very weak structured band in the near UV can be assigned to the delayed $S_{2} \rightarrow S_{0}$ fluorescence because of its approximate mirror symmetry to the $S_{0} \rightarrow S_{2}$ absorption band - see fig. 2. Two other components of the delayed luminescence are the delayed excimer fluorescence and the phosphorescence. The contribution of the delayed excimer fluorescence is estimated in the following section. The phosphorescence from biphenylene (cf. section 3.3) also contributed to the total delayed luminescence, but its contribution was small under the experimental conditions of the measurement of the spectrum in fig. 1.

\subsection{Delayed excimer fluorescence}

The intensity distribution of the delayed fluorescence in the spectral range of the $S_{1} \rightarrow S_{0}$ band significantly differs from that of the prompt $S_{1} \rightarrow S_{0}$ fluorescence. In the following we show that this difference 


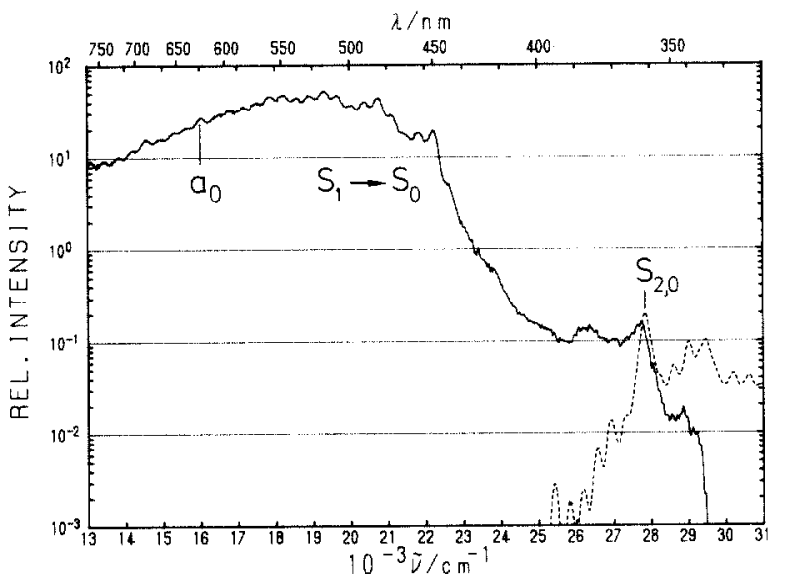

Fig. 2. Logarithmic plot $(-)$ of the delayed-luminescence spectrum of fig. 1 and of the absorption spectrum (---) of biphenylene in 3-methylpentane at $100 \mathrm{~K}$.

is likely to be due to the contribution of delayed excimer fluorescence to the total delayed fluorescence.

In principle, the determination of this contribution is simple when the spectrum of the monomer fluorescence has vibrational structure (as in the present case) and that of the excimer fluorescence is structureless, and when both spectra do not overlap completely. In this case the excimer band can be obtained by subtracting the spectrum of the prompt monomer fluorescence from that of the total delayed fluorescence, if both spectra have been normalized to equal intensity of the monomer fluorescence. In the present case, however, one meets several difficulties. Firstly, we did not measure the spectrum of the prompt $S_{1} \rightarrow S_{0}$ fluorescence at the same temperature, with the same spectral resolution and with complete elimination of polarization effects. Secondly, even if this spectrum were known, it may slightly differ from the spectrum of the monomer-like delayed $S_{1} \rightarrow S_{0}$ fluorescence due to TTA. Because of the short lifetime of $S_{1}$ (240 ps [11]), the two molecules in the $\mathrm{S}_{1} \ldots \mathrm{S}_{0}$ complex resulting from TTA cannot diffuse far apart. Hence the monomer-like delayed $\mathrm{S}_{1} \rightarrow \mathrm{S}_{0}$ fluorescence is always emitted by pairs of interacting molecules. The best one can do in this situation is to compare spectra in which the vibrational structure has been deliberately smoothed away.

In fig. 3 we compare the strongly smoothed spectra of the delayed luminescence (fig. 1) and of the

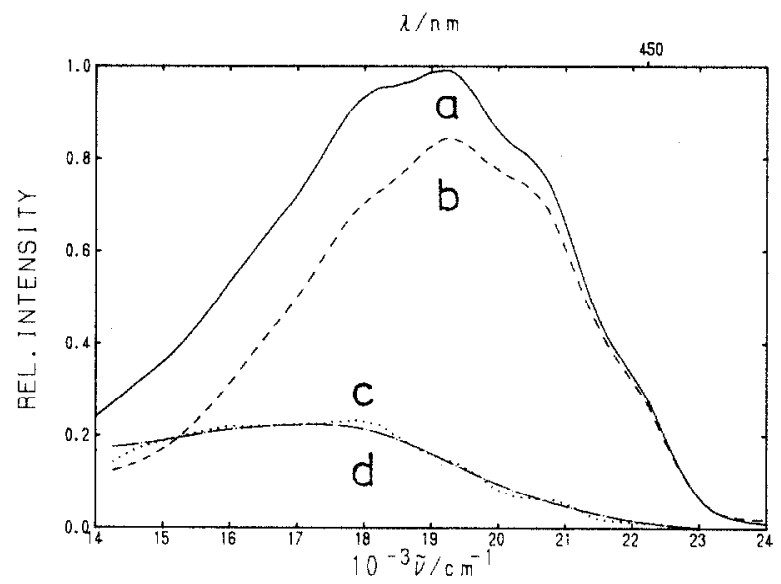

Fig. 3. Estimate of the contribution of delayed excimer fluorescence to the total delayed luminescence. (a) Smoothed spectrum (-) of the total delayed luminescence from biphenylene in isopentane/cyclopentane at $178 \mathrm{~K}$; (b) smoothed spectrum $(---)$ of the prompt $S_{1} \rightarrow S_{0}$ fluorescence from biphenylene in 3methyl-pentane at $130 \mathrm{~K}$; (c) difference spectrum (...) of the spectra (a) and (b); (d) smoothed difference spectrum (--).

prompt fluorescence (fig. 3 of ref. [4]). The two spectra are normalized to equal intensity on the right side, that is, in the spectral range of dominant or exclusive monomer-like delayed luminescence. The difference spectrum of the two spectra should be roughly equal to the spectrum of the delayed excimer fluorescence (we neglect the contribution of the phosphorescence at wavenumbers $\$ 16500 \mathrm{~cm}^{-1}$ ). According to this subtraction, $\approx 20 \%$ of the observed delayed fluorescence are delayed excimer fluorescence. The following points speak for this assignment.

(a) TTA does not require a sandwich conformation of the two interacting molecules in $T_{1}$. Therefore, after TTA in general a reorientation of the interacting molecules in the complex $S_{1} \ldots S_{0}$ is necessary for the formation of an excimer with a sandwich conformation. An appreciable formation of the excimer is possible only if the condition

$\tau_{\text {or }} \lesssim \tau_{1}$,

is satisfied, there $\tau_{\mathrm{or}}$ is the longest orientational relaxation time and $\tau_{1}$ the lifetime of biphenylene in $S_{1}$. With $\tau_{\text {or }}=v_{\mathrm{s}} \eta / k_{\mathrm{B}} T[10,4]$, with the effective volume $v_{\mathrm{s}} \approx 5.4 \times 10^{-23} \mathrm{~cm}^{3}$ of biphenylene in $\mathrm{S}_{1}$ [4], and with the viscosity $\eta \approx 1.6 \times 10^{-2} \mathrm{P}$ of isopentane/cyclopentane at $T=178 \mathrm{~K}[9]$, we obtain $\tau_{\mathrm{or}} \approx 35 \mathrm{ps}<$ 
$\tau_{1} \approx 240$ ps [11]. Thus, efficient secondary formation of an excimer should be possible.

(b) Typically the maximum ( $\tilde{\nu}_{\max }^{\mathrm{e}}$ ) of an excimer fluorescence band is red-shifted relative to the 0,0 transition $\left(\tilde{\nu}_{0,0}^{\mathrm{m}}\right)$ of the monomer fluorescence by $\Delta \tilde{\nu}_{\mathrm{m}, \mathrm{c}}=\tilde{\nu}_{0, \mathrm{o}}^{\mathrm{m}}-\tilde{\nu}_{\max }^{\mathrm{e}} \approx 6500 \pm 500 \mathrm{~cm}^{-1}$. For example, the spectra of the delayed fluorescence from three typical aromatic hydrocarbons in methylcyclohexane at $193 \mathrm{~K}$ yielded the following values of $\Delta \tilde{\nu}_{\mathrm{m}, \mathrm{e}}: 6400$ $\mathrm{cm}^{-1}$ for pyrene [12], $6600 \mathrm{~cm}^{-1}$ for 1,2-benzanthracene [12], and $6900 \mathrm{~cm}^{-1}$ for napthalene [13] (note, firstly, that $\tilde{\nu}_{\text {max }}^{\mathrm{e}}$ in general increases with increasing temperature and, secondly, that many literature values of $\tilde{\nu}_{\max }^{\mathrm{e}}$ (table 7.4 in ref. [14]) do not refer to corrected spectra on a wavenumber scale). For biphenylene, with $\tilde{\nu}_{0,0}^{\mathrm{m}} \approx 23900 \mathrm{~cm}^{-1}$ [4] and with $\tilde{\nu}_{\max }^{\mathrm{e}} \approx 17000 \pm 1000 \mathrm{~cm}^{-1}$, follows $\Delta \tilde{\nu}_{\mathrm{m}, \mathrm{c}} \approx 6900 \pm$ $1000 \mathrm{~cm}^{-1}$. Thus, with respect to its maximum, the spectrum of the excimer fluorescence from biphenylene would be normal.

(c) The relative intensity of a delayed excimer fluorescence at low solute concentration depends on six parameters: the probability $p$ for the formation of an excimer after formation of $S_{1}$ by TTA, the rate constant $k_{\mathrm{d}}$ for dissociation of the excimer, and the rate constants $k_{\mathrm{r}}^{\mathrm{m}}, k_{\mathrm{r}}^{\mathrm{e}}, k_{\mathrm{n}}^{\mathrm{m}}, k_{\mathrm{n}}^{\mathrm{e}}$ for the radiative (r) and nonradiative $(\mathrm{n})$ decay of the monomer $(\mathrm{m})$ and the excimer (e). When $k_{\mathrm{d}} \ll k_{\mathrm{r}}^{\mathrm{e}}+k_{\mathrm{n}}^{\mathrm{e}}$, the ratio $r_{\mathrm{e}, \mathrm{m}}=$ $I_{\mathrm{df}}^{\mathrm{e}} / I_{\mathrm{df}}^{\mathrm{m}}$ of the integrated intensities $I_{\mathrm{df}}^{\mathrm{e}}$ of the delayed excimer fluorescence and $I_{\mathrm{df}}^{\mathrm{m}}$ of the delayed monomer fluorescence is given by

$r_{\mathrm{e}, \mathrm{m}} \approx p \phi_{\mathrm{f}}^{\mathrm{e}} /(1-p) \phi_{\mathrm{f}}^{\mathrm{m}}$,

where $\phi_{\mathrm{f}}^{\mathrm{e}}=k_{\mathrm{r}}^{\mathrm{e}} /\left(k_{\mathrm{r}}^{\mathrm{e}}+k_{\mathrm{n}}^{\mathrm{e}}\right)$ and $\phi_{\mathrm{f}}^{\mathrm{m}}=k_{\mathrm{r}}^{\mathrm{m}} /\left(k_{\mathrm{r}}^{\mathrm{m}}+k_{\mathrm{n}}^{\mathrm{m}}\right)$ are the respective fluorescence quantum yields. With pyrene in methylcyclohexane at $193 \mathrm{~K}[12] r_{\mathrm{e}, \mathrm{m}} \approx 1.7$ is observed, and from the values of $k_{\mathrm{r}}^{\mathrm{m}}, k_{\mathrm{r}}^{\mathrm{e}}, k_{\mathrm{n}}^{\mathrm{m}}, k_{\mathrm{n}}^{\mathrm{e}}$ (table 7.3 in ref. [14]) follows $p \approx 0.6$. If we assume for biphenylene a slightly smaller value, $p \approx 0.5$, and $k_{\mathrm{d}} \ll k_{\mathrm{r}}^{\mathrm{e}}+k_{\mathrm{n}}^{\mathrm{e}}$, then from $r_{\mathrm{e} . \mathrm{m}} \approx 0.25$ follows $k_{\mathrm{r}}^{\mathrm{e}} / k_{\mathrm{n}}^{\mathrm{e}} \approx$ $0.25 k_{\mathrm{r}}^{\mathrm{m}} / k_{\mathrm{n}}^{\mathrm{m}}$. Since $k_{\mathrm{r}}^{\mathrm{m}}=1.0 \times 10^{6} \mathrm{~s}^{-1}$ [15] is unusually small, one may assume $k_{\mathrm{r}}^{\mathrm{e}} \geqslant k_{\mathrm{r}}^{\mathrm{m}}$; with this assumption we finally obtain $k_{\mathrm{n}}^{\mathrm{e}} \geqslant 4 k_{\mathrm{n}}^{\mathrm{m}} \approx 2 \times 10^{10} \mathrm{~s}^{-1}$. This is a reasonable result when internal conversion is the dominant nonradiative decay process of the excimer, since internal conversion in general becomes faster with a decreasing energy gap between the upper and the lower electronic state. Apart from inter- nal conversion, in principle formation of photodimers also contributes to the nonradiative decay of excimers. However, this contribution can be considered as negligible because of the observed relative photostability of the sample at $178 \mathrm{~K}$.

In conclusion, on the basis of the available data, the assignment of part of the delayed fluorescence to delayed excimer fluorescence is reasonable, but additional experiments are desirable. Firstly, the spectra of delayed and prompt fluorescence from biphenylene should be measured under the same experimental conditions and with the same spectral resolution. Secondly, a necessary condition for the assignment of part of the delayed luminescence to delayed excimer fluorescence is that, above the origin band of the phosphorescence, the decay curve of the delayed luminescence does not depend on the observation wavenumber; this should be confirmed experimentally. Thirdly, by choosing a lower temperature, it should be possible to realize the condition $\tau_{\text {or }}>\tau_{1}$ for the measurement of delayed fluorescence from biphenylene. Under this condition, the contribution of delayed excimer fluorescence to the total delayed fluorescence should be very small. Fourthly, at higher temperatures and under conditions of dominant triplet decay due to TTA, triplet-sensitized formation of photodimers of biphenylene should be observed. Finally, since the observation of prompt excimer fluorescence from biphenylene is practically impossible, the best way to demonstrate the ability of biphenylene to form an excimer would be the synthesis of a suitable biphenylenophane ${ }^{\# 1}$ and the measurement of its prompt fluorescence at low temperatures.

\subsection{Delayed $S_{2} \rightarrow S_{0}$ fluorescence}

The observation of a delayed $S_{2} \rightarrow S_{0}$ fluorescence is not surprising when $S_{2}$ is accessible by TTA [12]. In connection with ref. [4], the relative quantum yield $r_{\mathrm{df}}$ of this delayed fluorescence has been of interest. In the calculation of $r_{\mathrm{df}}$ we have to take account of the delayed $S_{2} \rightarrow S_{0}$ fluorescence hidden under the much stronger delayed $S_{1} \rightarrow S_{0}$ fluorescence and of the contribution of delayed excimer fluores-

\footnotetext{
\#1 A biphenylenophane is a sandwich pair of two biphenylene molecules that are linked by two or more alkane chains $-\left(\mathrm{CH}_{2}\right)_{n^{-}}$, typically with $n=2,3$, or 4 .
} 
cence. We compare the spectrum of the delayed fluorescence in fig. 2 with the spectra of the prompt emission and the hypothetical prompt $S_{2} \rightarrow S_{0}$ fluorescence in fig. 5 of ref. [4]. As representative wavenumbers we choose $22200 \mathrm{~cm}^{-1}$, where in both spectra the $S_{1} \rightarrow S_{0}$ fluorescence dominates, and 26200 $\mathrm{cm}^{-1}$, where the reabsorption of delayed $S_{2} \rightarrow S_{0}$ fluorescence is negligible. This comparison yields $r_{\mathrm{df}}^{\prime} \approx$ $0.5 r_{\mathrm{pf}} \approx 2 \times 10^{-3}$, where $r_{\mathrm{pf}}$ is the corresponding intensity ratio of the prompt fluorescence. By taking into account that the relative probability for the observation of monomer-like delayed $\mathrm{S}_{1} \rightarrow \mathrm{S}_{0}$ fluorescence is $1-p$, we obtain with $p \approx 0.5$ the final value $r_{\mathrm{df}} \approx(1-p) r_{\mathrm{dr}}^{\prime} \approx 1 \times 10^{-3}$. This value has been used in ref. [4] for the estimate of a safe lower limit $\phi_{2 f}^{f} \approx 2 \times 10^{-7}$ of the quantum yield of the prompt $S_{2} \rightarrow S_{0}$ fluorescence. $\phi_{2 f}^{l}$ may be too low on the following grounds. Firstly, the assumption, that in the singlet channel of TTA the generation of $\mathrm{S}_{2}$ is the dominant primary process, may not be justified. For example, the direct creation of vibronic states $S_{1,1,}$ by TTA is possible, and if $S_{3}\left(A_{8}\right)$ [15] is accessible by TTA, the internal conversion $S_{3} \rightsquigarrow S_{1}$ might bypass $S_{2}$. Secondly, relative to $S_{2.0}\left(\approx 27850 \mathrm{~cm}^{-1}\right)$, in TTA an excess energy $2 E\left(\mathrm{~T}_{1,0}\right)-E\left(\mathrm{~S}_{2,0}\right) \approx h c \times 4600$ $\mathrm{cm}^{-1}$ is available (for the value of $E\left(T_{1.0}\right) \mathrm{cf}$. section 3.3 ). Therefore, if one assumes that this excess energy reappears with equal probability as vibrational energy in the $S_{2}$ molecule and in the $S_{0}$ molecule, TTA should yield $S_{2}$ with an average vibrational energy of $h c \times 2300 \mathrm{~cm}^{-1}$; this would correspond to a prompt fluorescence excited at $30100 \mathrm{~cm}^{-1}-$ in contrast to the excitation wavenumber $\approx 28500 \mathrm{~cm}^{-1}$ [4] of the prompt fluorescence. Since in general intramolecualr vibrational relaxation becomes faster with increasing vibrational energy [16], one should expect for $S_{2}$ generated by TTA a lower fluorescence quantum yield than for $S_{2}$ generated by direct $S_{0} \rightarrow S_{2}$ excitation $\# 2$. Thus $\phi_{2 \mathrm{f}}^{\ell} \approx 2 \times 10^{-7}$ should indeed be a reliable lower limit.

\#2 As a referee has pointed out to us, this argument implies the assumption that the emitting molecules have no memory of their excitation route. If the lifetime of $S_{2}$ of biphenylene in a liquid solution is of the order of 8 ps [15], this assumption seems to be reasonable.

\subsection{Phosphorescence and triplet lifetime}

For the measurement of the phosphorescence spectrum of biphenylene, the relative contribution of TTA to the total triplet decay had to be strongly reduced. This was achieved by three changes in the experimental conditions: (a) The diameter of the laser beam in the sample was chosen as large as possible ( $\approx 1 \mathrm{~mm}$ ). (2) The excitation power was reduced to less than $1 / 10$ of that used for the measurement of the spectrum of the delayed fluorescnece. (3) A lower temperature was chosen $(150 \mathrm{~K}, \eta \approx 4 \mathrm{cP})$.

Under these experimental conditions, the contribution of delayed fluorescence to the total delayed luminescence at $16000 \mathrm{~cm} \quad$ ' was $\approx 50 \%$. In fig. 4 we show the phosphorescence spectrum of biphenylene; it is the difference spectrum of two spectra measured with low and with high excitation power and normalized to equal intensity of the delayed fluorescence. One might be tempted to assign the origin band $\mathrm{a}_{0}$ of this spectrum at $16020 \mathrm{~cm}^{-1}$ to the 0,0 transition of the phosphorescence. However, since $T_{1}$ is of $g$ symmetry, the 0,0 transition is symmetry forbidden. With the axis notation of fig. 4 , the space wavefunction of $T_{1}$ is of the symmetry $B_{3 g}[5,17]$. There can be no doubt about the symmetry of $T_{1}$, since there is no other triplet state close to $T_{1}$ (according to INDO/S $\mathrm{CI}$ calculations [17], $\mathrm{T}_{2}\left(\mathrm{~B}_{\mathrm{lu}}\right)$ is $\approx 7000 \mathrm{~cm}^{-1}$ above

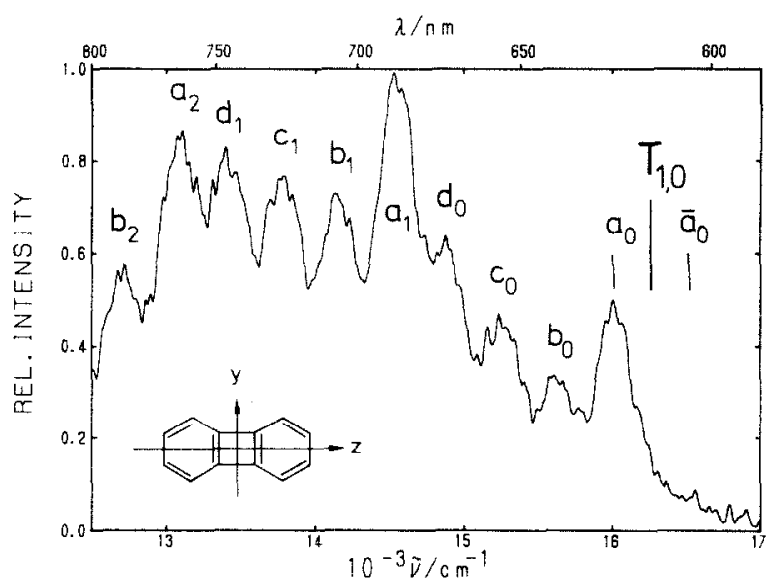

Fig. 4. Corrected spectrum of the phosphorescence from biphenylene in isopentane/cyclopentane at $150 \mathrm{~K}$. For the assignment of the vibronic bands of. table $1 . \vec{a}_{0}$ marks the position of the origin band of the oxygen-induced absorption of biphenylene [1]. 
$\left.T_{1}\left(B_{3 g}\right)\right)$. The three triplet substates are $T_{1 x}\left(A_{g}\right)$, $\mathrm{T}_{1, y}\left(\mathrm{~B}_{1 \mathrm{~g}}\right)$, and $\mathrm{T}_{1 z}\left(\mathrm{~B}_{2 \mathrm{~g}}\right)$ [18]. If the coupling non-totally symmetric vibration is of $b_{2 u}$ symmetry as in the case of the $S_{1} \rightarrow S_{0}$ fluorescence, then the emitting substate can be either $\mathrm{T}_{1 x}$ or $\mathrm{T}_{1 v} ; \mathrm{T}_{1 x}$ would yield a $y$ polarized phosphorescence, and $\mathrm{T}_{1 y}$ an $x$-polarized.

The $b_{2 u}$ vibration with the lowest energy is $\nu_{41}\left(b_{2 u}\right)=212 \mathrm{~cm}^{-1}[19]$. The difference between the origin band $a_{0}$ of the phosphorescence at 16020 $\mathrm{cm}^{-1}$ and the origin band $\overline{\mathrm{a}}_{0}$ of the oxygen-induced $\mathrm{S}_{0} \rightarrow \mathrm{T}_{1}$ absorption at $\approx 16530 \mathrm{~cm}^{-1}$ roughly corresponds to $2 \nu_{41}\left(b_{2 u}\right)$ (note that the value $16130 \mathrm{~cm}^{-1}$ deduced by Evans [1] refers to the onset and not to the first maximum of the spectrum ). Therefore we assign the origin band $\mathrm{a}_{0}$ of the phosphorescence to the vibronic transition $T_{1,0} \rightarrow S_{0, \nu 41}$ and the origin band of the oxygen-induced absorption to $\mathrm{S}_{0,0} \rightarrow$ $\mathrm{T}_{1, \nu 41}$.

The vibrational structure of the phosphorescence spectrum can be analyzed in a similar way as that of the $S_{1} \rightarrow S_{0}$ fluorescence in the preceding paper - see table 1. The main differences are as follows: Firstly, there is no evidence for the contribution of other $b_{2 u}$ vibrations than $\nu_{41}$. Secondly, apart from $\nu_{7}\left(\mathrm{a}_{\mathrm{g}}\right)$, also

Table 1

Vibrational assignment of the phosphorescence spectrum of biphenylene in fig. 4 . The wavenumbers $\tilde{\nu}$ of the vibronic contour bands were determined with an accuracy of $\pm 10 \mathrm{~cm}^{-1} . \nu_{\text {vib }}$ is the vibrational assignment relative to the false origin $a_{0}: \nu_{4}\left(a_{g}\right)=$ $1462 \mathrm{~cm}^{-1}, \nu_{6}\left(\mathrm{a}_{\mathrm{g}}\right)=1166 \mathrm{~cm}^{-1}, \nu_{7}\left(\mathrm{a}_{\mathrm{g}}\right)=1105 \mathrm{~cm}^{-1}, \nu_{9}\left(\mathrm{a}_{\mathrm{g}}\right)=$ $765 \mathrm{~cm}^{-1}, \nu_{10}\left(\mathrm{a}_{\mathrm{g}}\right)==395 \mathrm{~cm}^{-1}[19] ; \Delta \tilde{\nu}=\tilde{\nu}-\tilde{\nu}\left(\mathrm{a}_{0}\right)-\nu_{\mathrm{vib}}$

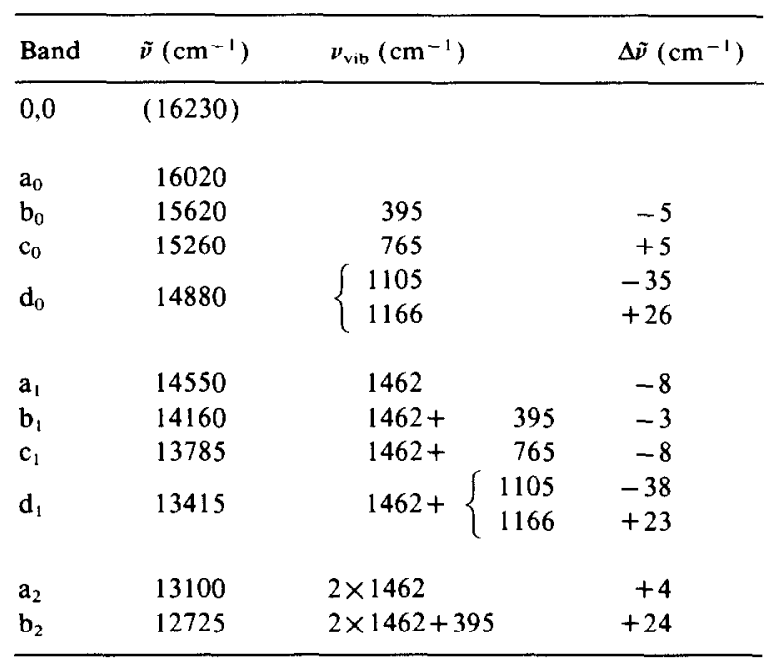

the neighbouring $\nu_{6}\left(\mathrm{a}_{\mathrm{g}}\right)$ seems to be active - cf. table 1. Thirdly, the $T_{1.0} \rightarrow S_{0, v_{4} 1}$ band is observed.

The second and the third differences can be rationalized in terms of bond-order changes. Calculated $\mathrm{CC}$ bond-order changes in $T_{1}$ relative to $S_{0}[17]$ are on the average by $22 \%$ smaller than those in $S_{1}$ relative to $S_{0}$ [15] (cf. table 4 in the preceding paper). Thus changes of $\mathrm{CC}$ bondlengths should be less important for the intensity distribution of the phosphorescence spectrum than for that of the fluorescence spectrum. This is also borne out by the intensity distribution in corresponding progressions of both spectra; for example, in the phosphorescence $a_{1}$ is the strongest vibronic transition, and in the fluorescence $\alpha_{3}$.

The assignment of the spectrum in fig. 4 to the phosphorescence of biphenylene has been corroborated by the measurement of decay curves of the delayed luminescence at $20800 \mathrm{~cm}^{-1}$ (maximum of the uncorrected spectrum of the delayed fluorescence) and at $14550 \mathrm{~cm}^{-1}$ (strongest band of the phosphorescence) - see fig. 5 . Both decay curves exhibit small but significant deviations from a monoexponential decay. At $20800 \mathrm{~cm}^{-1}$ this deviation results from a not yet negligible contribution of TTA to the total triplet decay. At $14550 \mathrm{~cm}^{-1}$ the deviation results mainly from the background of delayed fluorescence. The final slopes of both curves correspond rather accurately to the expected ratio of $2: 1$ and yield as triplet lifetime of biphenylene $\tau=330 \pm 10 \mu \mathrm{s}$.

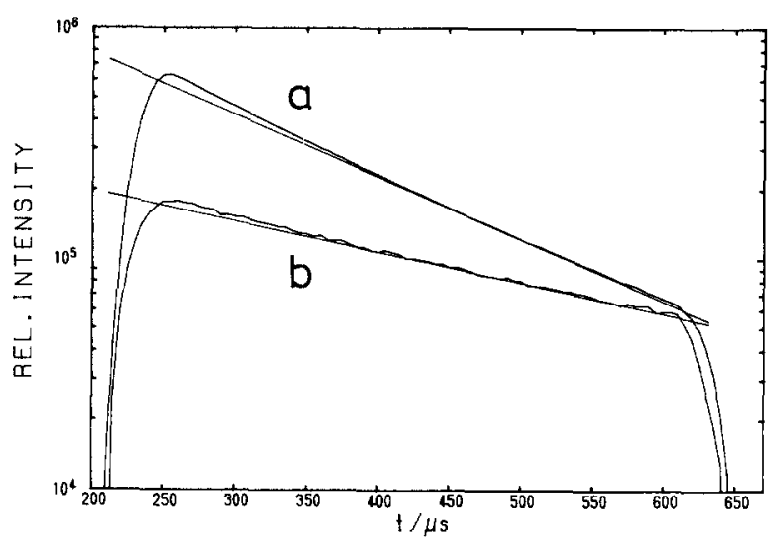

Fig. 5. Decay curves of delayed luminescence from biphenylene in isopentane/cyclopentane at $150 \mathrm{~K}:$ (a) at $20800 \mathrm{~cm}^{-1}$ (maximum of the uncorrected spectrum of the monomer-like delayed $S_{1} \rightarrow S_{0}$ fluorescence); (b) at $14550 \mathrm{~cm}^{-1}$ (maximum of the phosphorescence. 
Our value of the triplet lifetime is greater than that obtained from the decay of the triplet-triplet absorption of biphenylene in cyclohexane at room temperature $(\approx 100 \mu \mathrm{s}[2])$. This discrepancy may result from a genuine temperature dependence of the intrinsic triplet lifetime of biphenylene. However, we made no attempt to systematically investigate the temperature dependence of the triplet lifetime.

\subsection{Summary and conclusions}

(1) Delayed monomer-like $S_{1} \rightarrow S_{0}$ fluorescence from biphenylene is the dominant component ( $\approx 80 \%$ ) of the sensitized delayed luminescence from a solution of biphenylene and $\mathrm{N}$-methylcarbazole in isopentane/cyclopentane at $178 \mathrm{~K}$.

(2) The second strong component of the delayed luminescence $(\approx 20 \%)$ is assigned to delayed excimer fluorescence from biphenylene.

(3) The very weak delayed luminescence in the near UV is interpreted as delayed $S_{2} \rightarrow S_{0}$ fluorescence from biphenylene.

(4) At low triplet concentration of biphenylene, the phosphorescence from biphenylene is the dominant delayed luminescence below $16500 \mathrm{~cm}^{-1}$.

(5) The first band of the phosphorescence spectrum at $16020 \mathrm{~cm}^{-1}$ is a false origin. If the phosphorescence is induced by $a b_{2 u}$ vibration, the best value of the triplet energy of biphenylene is $E\left(\mathrm{~T}_{1,0}\right)$ $\approx h c \times 16230 \mathrm{~cm}^{-1}$.

(6) At $150 \mathrm{~K}$, the triplet lifetime of biphenylene is $\approx 330 \mu \mathrm{s}$.

\section{Acknowledgement}

We thank Professor A. Weller for support, Mr. M. Niemeyer for the preparation and purification of bi- phenylene, Mr. H. Lesche for the preparation of samples, Dr. B. Dick for discussions and for the communication of unpublished results, and Mr. M. Lindrum for valuable comments on the manuscript. We also thank a referee for valuable comments and suggestions.

\section{References}

[1] D.F. Evans, in: Optische Anregung Organischer Systeme, 2. Internationales Farbensymposium 1964 (Verlag Chemie, Weinheim, 1966) p. 590.

[2] C. Tetreau, D. Lavalette, E.J. Land and F. Peradejordi, Chem. Phys. Letters 17 (1972) 245

[3] K. Kimura, H. Ohno, K. Morikawa, Y. Hiramatsu and Y. Odaira, Bull. Chem. Soc. Japan 55 (1982) 2169.

[4] B. Nickel and J. Hertzberg, Chem. Phys. 132 (1989) 219.

[5] F. Peradejordi, C. Tetreau and D. Lavalette, J. Phys. Chem. 76 ( 1972$) 225$

[6] I.H. Munro, T.D.S. Hamilton, J.P. Ray and G.F. Moore, Phys. Letters 20 (1966) 386.

[7] B. Nickel and G. Roden, Chem. Phys. 53 (1980) 243.

[8] H.J. Haink and J.R. Huber. Chem. Phys. Letters 44 (1976) 117.

[9] H. Lesche and B. Nickel, unpublished results.

[10] F. Perrin, J. Phys. (Paris) 7 (1926) 390.

[11] H.-B. Lin and M. Topp, Chem. Phys. Letters 64 (1979) 452.

[12] B. Nickel, Helv. Chim. Acta 61 91978) 198.

[13] B. Nickel, unpublished results.

[14] J.B. Birks, Photophysics of Aromatic Molecules (WileyInterscience, New York, 1970).

[15] T. Elsaesser, F. Lärmer, W. Kaiser, B. Dick, M. Niemeyer and W. Lütke, Chem. Phys. 126 (1988) 405.

[16] S.M. Beck, D.E. Powers, J.B. Hopkins and R.E. Smalley, J. Chem. Phys. 73 (1980) 2019.

[17] B. Dick, unpublished results, private communication.

[18] S.P. McGlynn, T. Azumi and M. Kinoshita. Molecular Spectroscopy of the Triplet State (Prentice Hall, Englewood Cliffs, 1969).

[19] A. Girlando and C. Pecile, J. Chem. Soc. Faraday Trans. II 69 (1973) 818 . 